\title{
Telessaúde e COVID-19: estratégia de combate à pandemia e um novo caminho para o cuidado em saúde
}

\section{Telehealth and COVID-19: strategy to combat the pandemic and a new path for health care}

\author{
Lara Azevedo Teixeira' \\ Wellington Roberto Gomes de Carvalho ${ }^{2}$
}

Em março de 2020, a Organização Mundial da Saúde (OMS) declarou que a COVID-19, doença causada pelo coronavírus (CoV) da síndrome respiratória aguda grave 2 (SARS-CoV-2) tornou-se uma pandemia. Após três meses do surto e identificação da doença na cidade de Wuhan, na China, foram constatados mais de meio milhão de casos confirmados e 33.106 mortes no mundo [1]. À medida que a COVID-19 se propaga para outros continentes, crescem os esforços governamentais e das autoridades de saúde para minimizar os impactos desencadeados pela pandemia nos âmbitos social, econômico e político [2].

Diante desse grave cenário, diversas estratégias foram adotadas a fim de conter a transmissão do vírus, achatar a curva epidêmica, disponibilizando maior tempo para o desenvolvimento de tratamentos, para o ajuste da logística do sistema de saúde, bem como para testagem e manejo dos pacientes [3]. Nesse sentido, além das intervenções não farmacológicas, como distanciamento social, uso de máscaras e higiene das mãos, a telessaúde tem se demonstrado como um instrumento importante no combate da COVID-19 [4].

A telessaúde abrange o uso de tecnologias de informação e telecomunicação com a finalidade de promover cuidados à saúde à distância, difundir informações [5], bem como de oferecer serviços e atividades de treinamento para provedores assistenciais multidisciplinares [6]. Durante surtos de doenças infecciosas, como CoV da síndrome respiratória aguda grave (Severe Acute Respiratory Syndrome Coronavirus - SARS-CoV), CoV da síndrome respiratória do Oriente Médio (Middle East Respiratory Syndrome Coronavirus, MERS-CoV), Ebola e Zika, a telessaúde contribuiu para redução da transmissão viral por meio da implantação de um plano de ação composto pela monitoração remota de pacientes, teleconsultoria, bem como por teleconsultas, as quais possibilitaram a triagem dos casos e o atendimento de indivíduos isolados [4].

No entanto, apesar de registros do grande potencial que esse recurso apresentou no combate de epidemias anteriores, em muitos países, a regulamentação acerca da telessaúde ainda é escassa, principalmente, no que se refere à autorização, integração, ao reembolso dos serviços e à implantação da infraestrutura adequada [4]. No Brasil, apesar da existência de normativas a respeito da implementação da telessaúde, ainda não havia um marco regulatório devidamente consolidado no país [6].

\footnotetext{
${ }^{1}$ Aluna do Curso de Medicina, Faculdade de Medicina, Universidade Federal de Uberlândia, Uberlândia, MG, Brasil.

${ }^{2}$ Universidade Federal de Uberlândia, Instituto de Geografia, Curso de Saúde Coletiva, Uberlândia, MG, Brasil. E-mail: wrgcarvalho@ufu.br
}

Teixeira LA, de Carvalho WRG. Telessaúde e COVID-19: estratégia de combate à pandemia e um novo caminho para o cuidado em saúde Telehealth and COVID-19: strategy to combat the pandemic and a new path for health care. InterAm J Med Health 2020;3:e202003049. 
Apesar da criação do Programa Nacional Telessaúde e Redes e da Rede Universitária de Telemedicina (RUTE), que representam esforços com maior êxito voltados inserção da telessaúde no cenário nacional, ainda são muitos os desafios para a expansão e consolidação desse recurso no país. Um dos principais empecilhos à difusão da telessaúde, no Brasil, é a diversidade de resoluções propostas por diferentes órgãos com enfoques divergentes [5]. Até 2019, a telemedicina, vertente mais específica da telessaúde, era regulamentada pelo Conselho Federa de Medicina (CFM), o qual a restringia somente a comunicação audiovisual visando a assistência, educação e pesquisa em saúde, excluindo as possibilidades de consultas on-line, bem como de outros procedimentos e atendimentos à distância [6]

No entanto, com a rápida propagação da pandemia de COVID-19, o alto do número de casos infectados e a crescente demanda por cuidados na saúde desencadearam um cenário propício à implantação de novas estratégias para atenuar a pressão sobre o sistema de saúde [7]. Dessa forma, a telessaúde adquiriu grande relevância como estratégia de combate a COVID-19, haja vista sua capacidade de reduzir a circulação de pessoas nos estabelecimentos de saúde, evitando aglomerações, minimizar o risco de exposição tanto de profissionais da saúde, quanto de pacientes, além de possibilitar a liberação de leitos e vagas de atendimento hospitalar, priorizando os pacientes infectados [6]

Nesse sentido, o CFM, em março de 2020, incluiu no escopo da telemedicina, de forma temporária, enquanto durar a pandemia, os serviços de teleorientação, telemonitoramento e teleinterconsulta [8]. A partir dessa resolução, alterações na dinâmica do sistema de saúde têm sido evidenciadas, as quais possuem impacto sobre os profissionais da saúde, os pacientes e a população em geral. Dentre as mudanças, tem-se a aplicação da teletriagem, isto é, a análise de sinais e sintomas dos pacientes, por meio de plataformas virtuais e softwares específicos, com a finalidade de pré-selecionar aqueles indivíduos que apresentam um quadro de maior risco necessidade de encaminhamento ao hospital, evitando consultas desnecessárias e melhorando a qualidade assistencial [9].

O estabelecimento de "clínicas virtuais" também tem ganhado espaço no cenário de combate a COVID-19. Mediante a necessidade de reduzir a circulaçao pessoas e de priorizar o atendimento ao grupo de indivíduos infectados, muitos serviços de saúde adiaram e cancelaram consultas médicas, práticas clínicas, bem como cirurgias eletivas, porém tal medida pode não ser susten "clínicas virtuais" se apresentam como uma alternativ "ida que permitem a andise de exames de forma rema, o acompanamento de indvivus tex indivios com comorbidades e doenças preexistentes, os quais podem se encaixar no grupo de risco para a COVID-19 [6], e o monitoramento de pacientes recém liberados, por meio de aplicativos, smartphones, favorecendo a recomendaça de quarentena e atenuando a sobrecarga sobre departamentos de emergências [9].

Outro recurso facilitado pela telessaúde é a teleinterconsulta, a partir da qual profissionais da saúde podem trocar informações e opiniões entre si para auxílio diagnóstico ou terapêutico [9]. No contexto da COVID-19, essa medida tem permitido que médicos especialistas possam orientar outros trabalhadores na linha de frente, estabelecendo estratégias colaborativas mais eficientes, 0 que, além de melhorar os processos de decisão clínica, manter a capacidade de força de trabalho no sistema de saúde $[4,6]$.

Ademais, as tecnologias associadas à telessaúde têm se demonstrado relevantes năo apenas para a promoçăo de serviços, mas também para o empoderamento da população [10]. Por meio das tecnologias associadas a telessaúde, autoridades de saúde eórgãos governamentais the Twitter, WhatsApp e Facebook, que viablizamo acesso a informacões de qualida a atulização de dos sobre a pandsonia e qualidade, a atualizaçao de dados sobre a pandemia e o esclarecimento de possíveis dúvidas, fato que contribui também para atenuar a circulação de "fake news", bem como para aumentar engajamento da população no combate a pandemia da COVID-19 [6].

Em outros países, a telessaúde também tem demonstrado grande potencial no manejo da crise provocada pela rápida propagação da doença [11] Na China, o hospital da Universidade de Sichuan do Oeste Chines, em paceia com empresas de seviços telecomunicacão, elaborou uma rede multmod şcos telemedina, baseabrou uma rede multimodal de em tecnologia $5 \mathrm{G}$, voltada para a promoção de serviços médicos remotos, como clínicas virtuais, prescrições de medicamentos, realização e análise de exames, cuja implantação pode estar associada à baixa taxa de letalidade da COVID-19 na província de Sichuan $(0,55 \%)$ quando comparada àquela apresentada pela província de Hubei $(4,64 \%)$ e à taxa média mundial [12].
Com base no sucesso dessa estratégia na China, os Estados Unidos também apresentaram esforços para adoção da telessaúde no combate a COVID-19. Dentre esses, tem-se a flexibilização de leis no âmbito da saúde, como a Lei de Portabilidade e Responsabilidade do Seguro de Saúde, fato permite que provedores de cuidado usem telefones pessoais para assistir os pacientes, facilitando a implantação dos serviços no país [13]. Além disso, as companhias virtuais de assistência médica, como Teladoc e AmWell, têm se configurado como importantes instrumentos de comunicação entre profissionais e com pacientes, permitindo a realização de videoconferências seguras [12]. Outra iniciativa de grande relevância é a criação de programas eletrônicos de monitoramento de unidades de terapia intensiva, os quais viabilizam o acompanhamento remoto de dezenas de pacientes em hospitais distantes, reduzindo o risco de exposição e preservando a saúde dos profissionais [14]

Diante disso, a telessaúde se apresenta como uma estratégia eficaz no combate a COVID-19, haja vista u sua capacidade de promover triagem, cuidado e tratamento remotos, de garantir o monitoramento, vigilância e deteç̧ão dos casos infectados, bem como expandir as estratégias de prevenção da doença e aumentar o engajamento da população nesse processo [6]. No entanto, para que os serviços associados a esse recurso sejam explorados em sua potencialidade, é necessário que os países invistam não somente na infraestrutura precisas para a regulamentação dessas formas alternativas de cuidado à saúde [11]

Concluindo, a nova perspectiva acerca da telessaúde torna-se fortemente relevante frente ao cenário póspandemia, no qual esforços para a expansão dessa inevitável ferramenta permitirão ao sistema de saúde maximizar sua capacidade no que diz respeito ao saúde.

\section{REFERÊNCIAS}

1. Lee A, Morling J. COVID19: The need for public health in a time of emergency. Public Health. 2020;182:188-189. doi:10.1016/.puhe.2020.03.027

2. Chu D, AkI E, Duda S, Solo K, Yaacoub S, Schünemann $\mathrm{H}$, et al. Physical distancing, face masks, and eye protection to prevent person-to-person transmission of SARS-CoV-2 and COVID-19: a systematic review and meta-analysis. 9. $1-15$ doi:10.1016/ S0140-6736(20)31142-9 3. Carvalho WRG, Oliveira SV, Silva VP, Limongi JE. Distanciamento social: fôlego para ciência durante a pandemia de COVID-19 no Brasil. InterAm J Med Health. 2020;(3):1-6. doi:10.31005/iajmh.v3i0.113

4. Ohannessian R, Duong T, Odone A. Global Telemedicine mplementation and Integration Within Health Systems to Fight the COVID-19 Pandemic: A Call to Action. JMIR Public Health and Surveill. 2020;6(2):e18810. doi:10.2196/18810 5. Maldonado JMSV, Marques AB, Cruz A. Telemedicine: challenges to dissemination 2(suppl2):000155615 doi:10.1590/0102-311X00155615

6. Caetano R, Silva A, Guedes A, Paiva C, Ribeiro G, Santos $D$, et al. Desafios e oportunidades para telessaúde em tempos da pandemia pela COVID-19: uma reflexão sobre os espaços e iniciativas no contexto brasileiro. Cad Saúde Pública. 2020;36(5):e00088920. doi: 10.1590/0102-311X00088920

7. Contreras C, Metzger G, Beane J, Dedhia P, Ejaz A, Pawlik T. Telemedicine: Patient-Provider Clinical Engagement During the COVID-19 Pandemic and Beyond. I Gastrointest Surg. 2020:1-6. doi: 10.1007/s11605-020-04623-5 8. Conselho Federal de Medicina. Ofício CFM $n$ o 1.756/2020-COJUR. [internet]. $2020 \mathrm{Mar} 19$ [acesso em 22 Jun 2020]. Disponível em: http://portal.cfm. org.br/images/PDF/2020_oficio_telemedicina.

9. Moazzami B, Razavi-Khorasani N, Dooghaie Moghadam A, Farokhi E, Rezaei N. COVID-19 and telemedicine: Immediate action requiredformaintaining healthcareproviderswell-being. Clin Virol. 2020;126:104345.doi:10.1016/j.jcv.2020.104345

10. Fisk M, Livingstone A, Pit S. Telehealth in the Context of COVID-19: Changing Perspectives in Australia, the United Kingdom, and the United States. J Med Internet Res. 2020;22(6):19264. doi:102196/19264
Keshvardoost

Bahaadinbeigy 
K, Fatehi F. Role of Telehealth in the Management of COVID-19: Lessons Learned from Previous SARS, MERS, and Ebola Outbreaks. Telemed J E Health. 2020;10.1089/tmj.2020.0105. doi:10.1089/tmj.2020.0105

12. HongZ, LiN, LiD, Li J, LiB, XiongW, etal. TelemedicineDuring the COVID-19 Pandemic: Experiences From Western China. J Med Internet Res 2020;22(5):e19577.doi:10.2196/19577

13. Loeb A, Rao S, Ficke J, Morris C, Riley L, Levin A. Departmental Experience and Lessons Learned With Accelerated Introduction of Telemedicine During the COVID-19 Crisis. J Am Acad Orthop Surg. 2020;28(11):e469-e476. doi:10.5435/JAAOS-D-20-00380

14. Hollander J, Carr B. Virtually Perfect? Telemedicine for Covid-19. N Engl J Med. 2020;382(18):1679-1681. doi:10.1056/NEJMp2003539 